Joint Rheumatology Program, University of Athens, Athens; ${ }^{2}$ University of Ioannina, Ioannina; ${ }^{3}$ University of Crete, Heraklion; ${ }^{4}$ KAT Hospital, Athens; ${ }^{5}$ University of Thessaly, Larissa; ${ }^{6}$ Sismanoglio Hospital, Athens; ${ }^{7}$ Aristotle University, Thessaloniki; ${ }^{8} 401$ General Military Hospital; ${ }^{9}$ NIMTS Hospital; ${ }^{10}$ Private practice, Athens; ${ }^{11}$ Agios Andreas Hospital, Patras; ${ }^{12}$ Rheumatology Unit, Naval Hospital; ${ }^{13}$ Hygeia Hospital, Athens, Greece

Background: There are limited literature data regarding the characteristics of rheumatoid arthritis (RA) patients treated with biologic DMARD (bDMARD) monotherapy.

Objectives: To evaluate the disease and treatment characteristics of RA patients treated with bDMARD monotherapy.

Methods: Multicenter, cross-sectional RA epidemiological study in Greece (06/2015-05/2016, ERE RA Study Group). Demographics, disease characteristics, treatment and co-morbidity data were collected via a web-based platform Results: 1036 RA patients treated with bDMARDs were identified during the one year recruitment period (female: $82 \%$, mean age: $61.5 \pm 13$ years, mean disease duration: $12.5 \pm 8.9$ years, mean DAS28-ESR: $3.4 \pm 3.3)$. Among them, $26 \%(n=273)$ were receiving bDMARDs as monotherapy and $8 \%(n=23)$ of them had never tried conventional synthetic DMARDs (csDMARDs) before; The latter group $(n=23)$ compared to the csDMARD-exposed $(92 \%, n=250)$ group, had more often co-morbidities [cardiovascular disease (22\% vs. $8 \%, p=0.029)$, chronic hepatitis ( $13 \%$ vs. $3 \%, p=0.02)$, hypertension $(57 \%$ vs. $38 \%, p=0.08)$, COPD $(13 \%$ vs. $4.4 \%, p=0.07)$ ] or were active smokers ( $41 \%$ vs. $14 \%, p=0.001)$. csDMARD discontinuation was mainly due to adverse events (AEs, 58\%) followed by inadequate response (IR, 44\%). Compared to the cs- and b-DMARD combination therapy group, monotherapy treated patients were more frequently seropositive ( $64 \%$ vs. $57 \%, p=0.003$ ), had lower DAS28-ESR ( 3.2 vs $3.5, p=0.009)$ and were more likely to have discontinued csDMARDs for AEs ( $58 \%$ vs. $21 \%, \mathrm{p}<0.001)$ or IR $(44 \%$ vs. $27 \%, p<0.001)$. Tocilizumab $(22.3 \%$ vs. $12.7 \%, p<0.001)$ and rituximab $(19.8 \%$ vs. $13.5 \%, p=0.013)$ were utilized more often, whereas adalimumab less often $(8.8 \%$ vs. $14.8 \%, p=0.012)$ as monotherapy compared to as part of combination therapy.

Conclusions: In our large RA cohort, one out of four bDMARD treated patients, were receiving bDMARDs as monotherapy. Co-morbidities rather than RA characteristics influence the initial decision for bDMARD monotherapy whereas among those starting combination cs- and b-DMARD therapy, the majority discontinue csDMARDs due to AEs.

Acknowledgements: Grant support from the Hellenic Rheumatology Society and Professional Association of Rheumatologists.

Disclosure of Interest: None declared

DOI: 10.1136/annrheumdis-2017-eular.4390

\section{SAT0177 SAFETY EVENTS ARE SIMILAR WITH ABATACEPT VS PLACEBO TREATMENT IN RA: RESULTS FROM INTEGRATED DATA ANALYSIS FROM NINE CLINICAL TRIALS}

B. Soule $^{1}$, M. Hochberg ${ }^{2}$, D. Fleming ${ }^{1}$, A. Torbeyns ${ }^{1}$, T. Simon ${ }^{1}$, S. Banerjee ${ }^{1}$, M. Boers ${ }^{3} \cdot{ }^{1}$ Bristol-Myers Squibb, Princeton; ${ }^{2}$ University of Maryland, Baltimore, United States; ${ }^{3} \mathrm{VU}$ University Medical Center, Amsterdam, Netherlands

Background: Abatacept is a selective co-stimulation modulator that consists of the extracellular domain of human cytotoxic T lymphocyte-associated antigen-4 linked to the modified $\mathrm{Fc}_{\mathrm{c}}$ portion of human immunoglobulin and was approved initially for the treatment of RA in 2005. The integrated safety database for abatacept in adult RA comprises data from short- and long-term periods of 16 open-label and double-blind RA clinical trials, and combines studies of both IV and SC abatacept involving 7044 total patients. Of these, data from the 9 double-blind, placebo-controlled studies involving 4138 total patients were included in this analysis. The most recent overall safety analysis was performed in 2012 and reported IV and SC results separately.

Objectives: To provide an update of the overall abatacept safety profile based on data analysis from both IV and SC trials.

Methods: This evaluation included all patients with RA enrolled in 9 key doubleblind, placebo-controlled clinical trials of IV and/or SC abatacept. The safety analysis includes all serious adverse events (SAEs), as well as AEs of interest including infections, malignancies and autoimmune diseases.

Results: In total, 2653 patients were exposed to abatacept and 1485 received placebo. During the double-blind, controlled period, the mean (SD) duration of exposure was 10.3 (3.5) months for the placebo group and 10.8 (3.3) months for the abatacept group for a total exposure of 2357 and 1254 patient years, respectively (Table 1). The proportion of patients as well as incidence rates for death, SAEs, infections, malignancy and autoimmune diseases were similar between those treated with abatacept and placebo (Table 2).

Conclusions: In a large RA clinical trial database, safety events including deaths, serious infections, opportunistic infections, malignancies and autoimmune diseases occurred at similar frequencies and rates in the abatacept and placebo treatment groups.

Disclosure of Interest: B. Soule Employee of: Bristol-Myers Squibb, M. Hochberg Shareholder of: Theralogix LLC, Grant/research support from: $\mathrm{NIH}$, Consultant for: Bristol-Myers Squibb, EMD Serono Inc., Genentech/Roche, Novartis Pharma AG, Pfizer Inc, UCB Inc., D. Fleming Employee of: Bristol-Myers Squibb, A. Torbeyns Shareholder of: Bristol-Myers Squibb, Employee of: Bristol-Myers Squibb, T.
Table 1. Demographic Data

\begin{tabular}{lcc}
\hline & Abatacept $(\mathrm{N}=2653)$ & Placebo $(\mathrm{N}=1485)$ \\
\hline Mean age, yr (SD) & $51.7(12.4)$ & $51.4(12.3)$ \\
Female, \% & 79.1 & 79.7 \\
White, \% & 86.1 & 86.5 \\
NSAIDs, \% & 79.0 & 79.6 \\
Glucocorticoids, \% & 51.6 & 49.4 \\
Mean oral dose, mg (SD) & $7.5(8.1)$ & $9.1(9.1)$ \\
MTX, \% & 68.2 & 63.8 \\
Anti-TNF, \% & 6.4 & 5.5 \\
\hline
\end{tabular}

Table 2. Events During the Double-Blind, Placebo-Controlled Period

\begin{tabular}{lcclcc}
\hline & \multicolumn{2}{c}{ Abatacept $(\mathrm{N}=2653)$} & & \multicolumn{2}{c}{ Placebo $(\mathrm{N}=1485)$} \\
\cline { 2 - 3 } \cline { 6 - 6 } Outcome & $\mathrm{N}(\%)$ & $\mathrm{IR}(95 \% \mathrm{Cl})$ & & $\mathrm{N}(\%)$ & $\mathrm{IR}(95 \% \mathrm{Cl})$ \\
\hline Deaths & $12(0.5)$ & $0.5(0.3,0.9)$ & & $12(0.8)$ & $1.0(0.5,1.7)$ \\
SAEs & $331(12.5)$ & $14.8(13.3,16.5)$ & & $174(11.7)$ & $14.6(12.5,17.0)$ \\
Discontinued due to SAEs & $68(2.6)$ & & & $22(1.5)$ & \\
Infections & $1440(54.3)$ & $91.3(86.7,96.2)$ & & $761(51.6)$ & $90.8(84.4,97.4)$ \\
Serious infections & $41(1.5)$ & $1.7(1.2,2.3)$ & & $16(1.1)$ & $1.2(0.7,2.0)$ \\
Solid organ malignancies & $26(1.0)$ & $1.11(0.7,1.6)$ & & $12(0.8)$ & $1.0(0.5,1.7)$ \\
NMSC (combined) & $14(0.5)$ & $0.6(0.3,1.0)$ & & $5(0.3)$ & $0.4(0.1,0.9)$ \\
Autoimmune diseases & $198(7.5)$ & $8.5(7.3,9.8)$ & & $115(7.7)$ & $9.2(7.6,11.1)$ \\
COPD & $13(0.5)$ & $0.6(0.3,1.0)$ & & $2(0.1)$ & $0.2(0.0,0.6)$ \\
\hline
\end{tabular}

Simon Shareholder of: Bristol-Myers Squibb, Employee of: Bristol-Myers Squibb, S. Banerjee Shareholder of: Bristol-Myers Squibb, Employee of: Bristol-Myers Squibb, M. Boers Consultant for: Bristol-Myers Squibb

DOI: 10.1136/annrheumdis-2017-eular.2383

\section{SAT0178 PULMONARY INVOLVEMENT IN RHEUMATOID ARTHRITIS TREATED WITH BIOLOGIC}

E. Guerrero ${ }^{1}$, M.L. García ${ }^{1}$, E. Galindez ${ }^{1}$, E. Ruíz ${ }^{1}$, I. Torre ${ }^{1}$, L. Estopiñan ${ }^{1}$, O. Fernández ${ }^{1}$, A. Inchaurbe ${ }^{1}$, C. Perez $^{1}$, J. Blanco $^{1}$, I. Calvo ${ }^{1}$,

O. Ibarguengoitia ${ }^{1}$, N. Rivera ${ }^{1}$, I. Gorostiza ${ }^{2}$ on behalf of Rheumatology Service. ${ }^{1}$ Rheumatology Service; ${ }^{2}$ Research Unit, Basurto Hospital, Bilbao, Spain

Background: Use of biologic therapy (BT) has been determinant in Rheumatoid Arthritis' (RA) change of management and outcomes improvement for the last decade. Classic extraarticular manifestations are now uncommon, except for the pulmonary involvement, which can occur due to different causes and can complicate our patients' treatment and prognosis.

Objectives: The aim of this study is to evaluate the presence of pulmonary complication in RA patients under BT in our hospital, and assess its severity and the modifications required.

Methods: Review of clinical records of 208 RA patients receiving BT in the last 5 years (January 2012 to December 2016). 23 cases of preexisting lung disease for other causes have been excluded and finally 26 patients have been finally included. Demographic data, characteristics of RA, types of pulmonary involvement, evolution and changes in treatment have been collected. Statistical analysis were performed using SPSS v22.

Results: $73.1 \%$ are women, mean aged 59 years (31-80); $53.8 \%$ never smokers. They suffer from long term RA (median 176.92 months, SD 199.34); only 2 patientes have been recently diagnosed (less than a year). They are mainly seropositive (85\% RF positive) with positive CCP antibodies in $69.2 \%$, and with erosive disease in $70 \%$. $25 \%$ have other extraarticular manifestations (3 rheumatoid nodules, 4 cardiac involvement).

Half of the patients were in remission or low activity (DAS 28) at the time of pulmonary disease diagnosis, and the median of CRP was $0.52 \mathrm{mg} / \mathrm{dL}$ (SD 1.72). $90 \%$ had received Methotrexate and almost half of them Leflunomide; $30 \%$ had been treated with BT (50\% TNF alpha inhibitors).

Intersticial lung disease (ILD) was the most frequent pulmonary involvement $(57.7 \%)$ and non-specific intersticial pheumonia (NSIP) the most prevalent pattern $(>60 \%)$. We also found obstructive pulmonary disease $(11.5 \%)$ and vascular involvement $(7.7 \%) .40 \%$ of the patients had a normal radiograph (all of them a pathological CT)

Treatment was modified in $53.8 \%$ of the cases (synthetic DMARD was kept in $68 \%$ and $\mathrm{BT}$ in $64 \%$ ).

The average time of evolution of pulmonary involvement is 37.85 months (1-156). $80 \%$ of the patients kept stable or improved from their arthritis and also from respiratory disease. Only one received a lung trasplant and another one died.

We haven't found an association between different types of pulmonary involvement and the different variables analyzed in the study. We didn't show significant differences in prognosis related to pulmonary disease distinct patterns; up to $80 \%$ of patients with ILD stabilize or improve.

Conclusions: Prevalence of pulmonary disease in our experience in RA patients under BT is similar to prevalence in other observational studies $(10-20 \%)$, because diagnosis here is due to casual detection in a routine chest X-ray or for clinical suspicion for respiratory symptoms (cough, dyspnea, ...). The evolution has been good perhaps for the high prevalence of NSIP, which requires less therapeutic intervention. Protocols for systematic search of lung disease in RA patients seem essential for its proper diagnosis, and there is a need for further research in pathogenesis and management of this complication. 